\title{
CONSTRUIR CONVIVENCIA EN UN PAIS EN MEDIO DEL CONFLICTO: UNA EXPERIENCIA PEDAGÓGICA ALTERNATIVA
}

Gema Trujillo Pérez Neiva. Huila- Colombia Milena Trujillo Perdomo Neiva. Huila-Colombia $\left.{ }^{*}\right)$

\section{ORIGEN DE LA EXPERIENCIA}

Producto de las investigaciones realizadas desde la Especialización en Comunicación y creatividad para la docencia, una de ellas : "Transformación de la práctica pedagógica en Neiva entre 1960 y 1990" y otra sobre "Desplazados por la violencia y no por violentos: el caso de la población desplazada hacia la ciudad de Neiva entre 1998-2000”; se advierte que ,la estructura de la Escuela sigue permeada por los rasgos de la Educación tradicional, descontextualizada de su entorno, ajena al reconocimiento de la realidad socio-cultural de la población desplazada, afectada por la guerra vivida en el país; dos maestras, inquietas por encontrar nuevos paradigmas y fruto de sus búsquedas, ,se unen por opción, a la experiencia pedagógica de la Escuela Popular Claretiana de Neiva y hoy presentan su reflexiones en torno al papel que ha jugado ésta, en la construcción de ciudadanía, en un sector excluido de la ciudad.

Para entender el origen de la experiencia es necesario ubicarse dentro de las tendencias pedagógicas en América Latina y el movimiento pedagógico colombiano dentro de la corriente del pensamiento de renovación educativa y cultural, a mediados de los 80s; que propuso además, pensar el rol del maestro como intelectual, cultural y político, en un espacio que permita articular la ética pedagógica, fundada sobre una política democrática y un saber pedagógico, como lo plantearon los gestores del movimiento pedagógico, en la declaración final del Congreso Pedagógico Nacional. (FECODE, 1987, p. 9-10).

Dicha discusión fue liderada desde la Federación, ,junto al esfuerzo y compromiso de ,maestros colombianos, con el propósito de transformar "una práctica educativa bancaria y domesticadora" por una educación que contribuya ,a liberar al hombre y a la sociedad y, según la

\footnotetext{
${ }^{(*)}$ Gema Trujillo Pérez, Magíster en Conflicto, Territorio y Cultura. Docente Escuela Popular Claretiana. Neiva. Huila- Colombia. E-mail: gematrujillop@gmail.com .

Milena Trujillo Perdomo. Magíster en Conflicto, Territorio y Cultura. Docente Escuela Popular Claretiana.

Neiva. Huila- Colombia. E-mail. milenatrujillo2004@yahoo.com.
} 
expresión de Paulo Freire, de la dependencia y de todo tipo de alineación; ,los gestores de la propuesta, reconocen también, que para la construcción de la propuesta, retomaron elementos de la Investigación- Acción Participativa que empezaba a emerger ,en Colombia. (POSADA, SIGNORI,1987, Pág. 6)

Además, ya desde la década de los 70 s, la comunidad Claretiana en Colombia, guiada por los principios de la corriente de una Educación Liberadora, inicia una experiencia pedagógica, en un colegio privado fundado en 1966, en barrios del Sur y del occidente de Bogotá, para niños y jóvenes en niveles de pobreza. Es de resaltar, que uno de los aspectos que contribuyó al logro de la propuesta a nivel global, fueron los procesos de formación continua de los educadores, como lo plantean en una de sus reflexiones sobre el papel de la educación en la estructura social, la situación de la educación en Colombia y temas que ayudaron a comprender los alcances y límites del trabajo educativo.

Tales reflexiones, advierten la necesidad de afectar la práctica ,y se acogen ,al método de la "enseñanza personalizada"; con el trascurso de los meses descubren las contradicciones y en la búsqueda de alternativas teóricas y metodológicas, coherentes con los objetivos propuestos, inician un ,proyecto de escuela Popular, orientado por lor principios del Movimiento impulsado por Celestin Freinet; dicha experiencia se suspende en este lugar, por distintas razones.

Es así, ,como en 1980, los promotores de Bosa, encuentran más favorables las condiciones para iniciar una obra educativa de carácter popular, en los barrios Surorientales de Neiva, a raiz de la invasión de Peñon redondo, en un sector marginal ubicado en la periferia ,de la ciudad, como receptor de población desplazada, proveniente de diferentes zonas de conflicto del país, principalmente del Tolima, Caquetá, Putumayo y de varios municipios del departamento del Huila; familias cansadas de la confrontación entre guerrillas y ejército colombiano, quienes llegan en búsqueda ,de empleo, o de oportunidades para sus hijos; lugar que posteriormente denominaron ,"Filodehambre”, nombre con el cual también asignaron a la ,experiencia pedagógica, como lo expresan en su primera publicación. (POSADA,SIGNORI,1987, p. 22)

Actualmente, continúa ubicada en el barrio Los Alpes, de la Comuna ocho de Neiva, lugar que sigue siendo receptor del mismo tipo de población, en condiciones de vulnerabilidad, pero además estigmatizado por el resto de la población. Ahora se conoce con el nombre de Escuela Popular Claretiana y a partir de la reestructuración educativa en el 2001, hace parte de la Institución Educativa Normal Superior de Neiva, cuyo propósito es el de formar maestros. 


\section{SU FUNDAMENTACIÓN TEÓRICA}

La experiencia se alimenta fundamentalmente de los aportes pedagógicos y didácticos de Celestín Freinet y de la propuesta de Educación Popular de Paulo Freire. Para Freinet, la pedagogía se entiende como:

un proceso educativo que satisface las necesidades y derechos de niñas y niños; la escuela se concibe como lugar de producción y éxito de un trabajo libremente escogido, organizado y recreado; en consecuencia, la educación se basa en la cooperación, que tiene como propósito, cambiar las relaciones de autoridad por relaciones horizontales de ayuda y solidaridad, donde, la organización del trabajo escolar contribuye al desarrollo de la personalidad tanto de estudiantes como a los adultos.

Una escuela del pueblo, implica situar los aprendizajes en el marco de una pedagogía cooperativa, que desarrolla un sistema económico y social, cuyos valores sean el espíritu crítico, la responsabilidad, la iniciativa, el apoyo mutuo, el desarrollo personal y la solidaridad. (FREINET, 1977, p. 49).

Donde, además, concibe al educador, como la persona que coordina, anima todas las actividades y despierta las motivaciones vitales de niños y niñas, sujeto que propone perspectivas, suscita la iniciativa, la responsabilidad, sustituye la imposición y competencia, por la realización de proyectos de interés y significación para los estudiantes; dicha percepción, exige del maestro una clara vocación de compromiso con los sectores excluidos de la sociedad, para transformarlos en protagonistas de su historia y en sujetos autónomos, capaces de tomar iniciativas.

De Paulo Freire, como educador comprometido con la transformación de la realidad social, se retoma su propuesta de:

Una Educación Popular Liberadora, la preocupación y responsabilidad por la formación de un hombre capaz de superar la captación ingenua y la posición pasiva ante su realidad; igualmente, postula el desarrollo de una actitud científica centrada en la búsqueda de explicaciones causales y en la comprensión histórica de los hechos dentro de la dinámica del pasado, presente y futuro. (Proyecto Educativo Institucional; 1997, p. 21).

La experiencia se inserta en un proyecto más amplio de educación popular, el cual toma los siguientes elementos. Se plantea que los sectores populares descubran su identidad de clase, perciban sus propios intereses y se organicen para defenderlos, como protagonistas de las transformaciones históricas. 
a) La tarea fundamental de la educación popular es la formación del hombre nuevo, libre de tendencias dominadoras, que lucha por la construcción de una sociedad dentro de un proyecto liberador.

b) Se propone un tipo de conocimiento que supere la captación ingenua de la realidad y desarrolle una actitud científica.

c) La educación popular se plantea la reapropiación crítica de la cultura, es decir el rescate de los saberes populares.

Apoyados en estos elementos, propusieron métodos y técnicas que recogen los contenidos que nacen de la realidad conflictiva y que se orientan hacia su transformación. (POSADA, 1987p. 128-131).

Con el transcurrir de los años en torno a la reflexión de la experiencia, con la lucha y defensa colectiva, se convirtió en una escuela Pública, abierta a las nuevas tendencias pedagógicas y políticas, donde se enfatiza en la necesidad de la apropiación crítica de la cultura, a través de métodos que recojan los contenidos nacidos de la realidad, se recupere la palabra y su capacidad creadora y no sigan sometidos a una cultura del silencio.

En consecuencia, el acto educativo no consiste en una trasmisión de conocimientos, sino en disfrutar la construcción de un mundo compartido; en donde se defiende el derecho del pueblo de ser sujeto y no objeto de investigación, por especialistas ajenos a él, se acoge, además, la necesidad de desarrollar la pedagogía de la pregunta.

Diversos autores han enriquecido el proyecto Educativo, entre ellos: Franco Tonucci, con su propuesta de investigación como alternativa a la enseñanza; Lev Vygotsky, con la tesis socioconstructivistas $\mathrm{y}$ en el campo de las áreas, especialmente en matemáticas, lenguaje y comunicación, Jerónimo Bruner, Josette Jolibert, Néstor García Canclini, Jesús Martín barbero, Noam Chomsky, entre otros. También pedagogos, que sus aportes han enriquecido la reflexión sobre la práctica de la escuela y han permitido redefinir el Proyecto Educativo Institucional.

Cada uno de estos autores, ha contribuido al proceso de construcción del proyecto educativo, el cual se inscribe en una práctica social y como tal, sujeta a la dinámica de los procesos sociales que son históricos y cambiantes.

El fundamento sólido inicial, estuvo en la inspiración ética y cristiana que orienta el quehacer educativo; en coherencia con esto, se debe vivenciar en la práctica escolar y social, los valores humanos de justicia, equidad, solidaridad, honradez y autenticidad, propuestos desde la 
corriente de Educación Liberadora, como como lo expusieron en un artículo en el congreso pedagógico nacional:

Se asume como educación cualificada, la que considera a los actores de la acción educativa como sujetos y protagonistas de los procesos de aprendizaje y producción de conocimientos y destrezas que parten de experiencias vitales y significativas. ,La formación integral, liberadora y autogestionaria, provoca el desarrollo pleno y autónomo de los sujetos, descubre valores y potencialidades, para enfrentar, críticamente, los retos que les plantean los cambios afectivos, sociales, culturales, científicos y tecnológicos. ,Este enfoque abre el camino a la "dimensión democrática" y de formación ciudadana que se experimenta en la relaciones fraternales y solidarias, en el respeto y valoración de las diferencias. (POSADA,SIGNORI,1987, p. 51)

\section{¿CÓMO SE CONCRETAN LOS POSTULADOS Y PROCESOS DE FORMACIÓN PARA LA PARTICIPACIÓN Y CONVIVENCIA EN LA ESCUELA POPULAR?}

Los principios que plantea la propuesta se operacionalizan en la vida cotidiana, a través de estrategias que forman "en y para la participación ciudadana” cuya intencionalidad pedagógica y política de la Escuela conlleva ,a la formación crítica del estudiante y de sus actores frente a su contexto, por ello, los gestores de la propuesta definen la pedagogía como: "un proceso intencional de liberación de la persona para que desarrolle sus potencialidades con libertad, responsabilidad y eficacia, además logre encaminar su vida a la transformación de su entorno”. ,(POSADA, SIGNORI, 2009, p. 51)

Desde el inicio hasta la actualidad se han implementado varias estrategias, entendidas como: un conjunto de actividades que se relacionan entre sí y se orientan a un objetivo concreto: la formación para la participación, proceso flexible desarrollado a largo plazo y que pretenden contribuir desde diferentes ámbitos a la construcción de una cultura democrática y, en definitiva, a la formación del individuo como ciudadano. (POSADA, SIGNORI, 2009, p. 51)

Las estrategias que fortalecen los procesos de participación en los tres actores niños, niñas, padres de familia y maestros son: el gobierno escolar entendido como un órgano de dirección compartida, facilita la participación de la comunidad educativa en la gestión general de la escuela través de diversos estamentos que la integran, el Plan anual como una planeación abierta, susceptible de ser revisada, recreada ante las nuevas coyunturas y necesidades de los agentes educativos. 


\section{LOS PROYECTOS DE AULA, COMO ESTRATEGIA QUE PROPICIA LA INVESTIGACIÓN. ESTOS SE DEFINEN COMO}

La mediación pedagógica y didáctica para hacer realidad los objetivos y estrategias formuladas en el plan anual, en un espacio más reducido como es el aula, enfocan su acción a la investigación; actividad esencial que facilita la identificación de fortalezas y problemas, su análisis y la búsqueda de propuestas de solución. A la vez, integran los aprendizajes y definen la pertinencia y globalización de los contenidos curriculares.

[...] El proceso de elaboración y desarrollo de estos proyectos, comprende cinco pasos: la organización inicial del trabajo, la investigación del medio y recolección de información, el procesamiento específico de contenidos, la asimilación de aprendizajes a través del arte; la socialización y evaluación; por último, la integración de la escuela con la comunidad.

[...] Considerada esta estrategia como un escenario vivo donde se intercambian saberes, afectos, intereses, valores, etc., la participación activa y organizada se torna imprescindible, que se evidencia en la propuesta metodológica en diferentes momentos: 1. Actualización del diagnóstico personal y familiar. 2.. Formulación del proyecto, 3. Organización cooperativa del trabajo, 4. Plan de investigación, 5. Procesamiento de la información y 6. Evaluación y socialización. (POSADA, SIGNORI, 2009, p. 59-69)

Se desarrollan también, unas estrategias que promueven el mejoramiento de las condiciones de vida y trabajo escolar en la Escuela que son los programas de nutrición y el de útiles escolares colectivos, los cuales, los desarrollan padres de familia, acompañados por los maestros. Éstas nacen fruto de la necesidad sentida por los diferentes actores, para garantizar la construcción colectiva de conocimientos, mejorar sus niveles de aprendizaje y además con el fin de favorecer el trabajo en el aula de los niños y niñas en condiciones de igualdad y para el desarrollo de valores cooperativos de respeto, responsabilidad y solidaridad.

Las diferentes estrategias, se implementan con la participación de los tres actores, con propósitos como: el mejoramiento de las condiciones de nutrición y de salud, la consolidación de hábitos alimenticios, la consecución de los recursos necesarios para el trabajo, el cuidado y buen de los mismos, y el logro de objetivos propuestos en los Proyectos de aula.

Todas las estrategias, implican procesos de planeación, organización, desarrollo y evaluación permanente de los procesos vividos por parte de los tres actores; incentivan la creación 
de ambientes de participación en diferentes niveles, el desarrollo de actividades grupales en horas extra escolares, a la vez, que favorecen la integración a procesos grupales y la valoración y el reconocimiento de sus actores, contribuyen con la continuidad y permanencia de la Experiencia.

\section{BALANCE ,DE LA EXPERIENCIA}

Trascurridos 38 años de la experiencia, y al realizar un balance general, se encuentra que dentro de los factores que han facilitado la implementación de la propuesta, es el haber contado con un equipo interno que asesoró y acompañó la práctica de los maestros; un equipo externo, que generó un trabajo sistemático, riguroso, de reflexión permanente sobre la práctica y de procesos de autoformación. También, contar con un grupo de maestros inquietos, comprometidos y convencidos en la necesidad de repensar el papel de la escuela y el rol del maestro.

Otro factor que favorece, la permanencia de la experiencia, cerca de cuatro décadas, es el contar con documentos que contienen una fundamentación teórica y metodológica, producto de la sistematización, donde se recoge la vivencia y testimonios, como base para la consolidación del proyecto pedagógico, el cual, se ha convertido en respuesta a una para la transformación del entorno y de una población con características y necesidades específicas, como lo plateaba Paulo Freire: "la necesidad de la transformación, de la reinvención del mundo en favor de las clases oprimidas" ( FREIRE; 1997, pág.90)

El tener un reconocimiento a nivel local, nacional e internacional, se convierte en referente pedagógico y de los procesos comunitarios; situación que conlleva a que los diferentes actores se interesen en conocer y entender el propósito y dinámica e involucrarse en los procesos, desde los mismos proyectos de aula. También, el mantener viva la memoria de la historia y sus protagonistas, a través de murales visibles y de un museo, que cuenta la historia por medio de fotografías, de objetos que representan los momentos y hechos significativos y que se sintetiza en una línea del tiempo.

Favorecer la construcción de conocimiento nuevo, útil y válido, ser respuesta a necesidades sentidas de niños, niñas y sus familias, convertir las diferentes disciplinas del conocimiento en el apoyo para la comprensión de las problemáticas priorizadas y buscar alternativas de solución de manera colectiva.

Respecto a los factores que dificultan la implementación, en primer lugar, las reformas a la educación, como lo expresa el colectivo de maestros en el libro la participación en la escuela, cuyo énfasis está en: 
...reducir la inversión social, privatizar la educación pública, centralizar la administración y el poder mediante la agrupación de instituciones escolares, aumentar la cobertura en detrimento de la calidad educativa, medidas que han afectado la autonomía, la participación de los actores en las decisiones, de gestión y la toma de ,decisiones, sin tener en cuenta el proceso de contrucción de un currículo y las formas organizativas propias, causando en unos, frustración y en otros, desconcierto. (POSADA, SIGNORI, 2009, p. 100)

Esto, producto de las políticas educativas y de los planes de los gobiernos acogidos a las tendencias neoliberales, de las últimas décadas y en consecuencia, la implementación de programas de formación dirigidos a los maestros que no conlleva a generar inquietud, ni motivación para comprender las nuebas realidades, ni la construcción de personas autónomas, con pensamiento crítico; por el contrario, tiende a estandarizar, a la masificación y competitividad de las instituciones y de individuos. Todo lo anterior ha generado, inestabilidad en la planta de maestros, cambio constante de educadores,y falta de divdersos apoyos que contribuya a comprender las nuevas realidades.

Se agudiza cada vez más, la situación socioeconómica de las familias, que se evidencia en las actividades diarias del rebusque y los altos índices de desempleo, y que repercute en los procesos de participación y desarrollo de las organizaciones, ya sea porque prefieren buscar soluciones de manera individual o porque no disponen del tiempo para los procesos de formación.

Pero, además, la cultura de la dependencia y la informalidad, características de los actores excluidos, dada la actitud pasiva o la falta de constancia, iniciativa y continuidad para resolver los problemas de manera colectiva, producto de cierta desorganización en la vida cotidiana del hogar, impiden procesos continuos y de mayor nivel de participación.

La experiencia por sí misma, exige un compromiso mayor por parte del maestro, pues éste, se convierte en el motor dinamizador de todos los procesos, y en consecuencia, se requiere que asuma espacios autoformación, dispuesto a trabajar en equipo, con apertura a la crítica, a la transformación y a la reflexión permanente sobre la práctica, situación, que no todos los educadores están dispuestos asumir.

Queda en evidencia, que mientras los educadores y educandos, comprendan, la urgencia de asumir el papel ,crítico como sujetos conocedores de las necesidades y, o problemáticas de la realidad, de las expectativas e interrogantes de los actores, ,de articular procesos de formación de manera integral, donde además, se logre articular la teoría con la práctica, que se gane conciencia 
del sentido de la participación en los distintos espacios, sólo así se concretará la valoración de sí mismos, elevar la autoestima y la realización personal.

\section{IMPACTO DE LA EXPERIENCIA}

Pese a las múltiples dificultades para sacar adelante la experiencia, la propuesta, contribuyó en la defensa de la calidad de vida, que se refleja, en la preocupación por, mejorar la vivienda, en adquirir los servicios públicos básicos, la adecuación y mejoramiento de la planta física de la escuela; la formación integral de agentes populares para asumir la gestión de pequeñas microempresas orientadas al desarrollo de programas de salud y nutrición.

Hoy, a pesar de las dificultades propias del país, además de mejorar el entorno, hay avances en la convivencia al interior de las familias, en un buen porcentaje, en la construcción de sus proyectos de vida para sus hijos, la valoración y cuidado del medio ambiente, en ofrecer otros espacios para la formación, en el manejo adecuado del tiempo libre, todo esto, a partir de las dinámicas que se generan desde la investigación en los proyectos de aula y las estrategias que promueve la escuela.

En coherencia con el fundamento inicial, respecto a la inspiración ética y cristiana, además de seguir fortaleciendo los valores humanos y sociales en la práctica escolar y social, se continúa con la valoración de las diferencias culturales y de género; la relación respetuosa con las demás personas, la creación de hábitos, de responsabilidad y autonomía frente al trabajo colectivo. ,

Se tiene conocimiento de casos de exalumnos, que logran terminar la secundaria, destacándose en el liderazgo estudiantil, y unos, que han tenido mejores condiciones terminan sus estudios universitarios, se han vinculado a instituciones y, u organizaciones, desempeñándose con un compromiso y sentido comunitario. Es de resaltar, también que la formación continua de maestras y maestros, aporta significativamente a nivel personal, profesional y familiar.

A lo largo de la experiencia, ha contribuido a los procesos de formación de otros maestros, el intercambio con otras instituciones educativas en todos los niveles, tanto a nivel local, como nacional y con ello a generar cambios y transformaciones significativas.

La reestructuración de las Instituciones educativas, implicó para la escuela, ampliar su radio de acción a todos los centros asociados con la Normal y con ello permear en la consolidación de una propuesta alternativa para fortalecer el Proyecto Educativo Institucional, que involucra a maestros en ejercicio y en procesos de formación a través de prácticas que la retroalimentan. 
Los avances en los ambientes al interior de las familias, los logros a nivel personal y profesional de los jóvenes y de los mismos maestros, los efectos en los diferentes campos educativos, reafirma la vigencia y pertinencia de la escuela y su experiencia; por ende, su compromiso con la Educación pública, frente a la crisis educativa generalizada, la agudización de los problemas intrafamiliares, en un país en medio de la incertidumbre y el conflicto.

\section{A MANERA DE CONCLUSIÓN:}

Los procesos que se llevan a cabo al interior de cada una de las estrategias adquieren sentido, en la medida en que se implementen en los sujetos una actitud crítica y comprometida con los cambios que las nuevas realidades exigen, se propicie la creación de ambientes pedagógicos, pues en ellos, la acogida, la confianza y el respeto a las diferencias, favorecen la construcción de tejidos comunicativos.

También, es la reflexión sobre la práctica, la que permite con una actitud crítica se reconstruyan conceptos, valores, métodos y a la vez se clarifiquen las motivaciones e intereses individuales frente a los objetivos de cada grupo u organización, junto con el análisis de los procesos vividos, siendo éste, el núcleo central de la evaluación, entendida como una oportunidad de reflexión y cambio.

Se constata con la implementación de las diferentes estrategias, que desde temprana edad niños y niñas empiezan a construir la semilla de convivencia a partir de la organización cooperativa que transversaliza la cotidianidad escolar a partir de la vivencia de valores, tales como: respeto, responsabilidad, autonomía y solidaridad. De igual manera, el desarrollo de la conciencia crítica y la formación ciudadana de los estudiantes se potencia a través de la investigación del entorno y las preguntas que le generan su cotidianidad.

La autonomía que se fomenta en la Escuela enriquece al individuo para que pueda sentirse miembro de un grupo y constituirse en sujeto de sus propios saberes, afectos, ilusiones y proyectos, además brinda orientacion y apoyo para generar espacios democráticos y favorecer ,los intercambios y las búsquedas compartidas, respetando las diferencias.

Los valores de solidaridad, equidad, cooperación y justicia se re-significan para configurar nuevas formas de convivencia y relaciones sociales en respuesta a deseos y sueños compartidos por los actores de la Escuela, de igual manera, la práctica de los valores conlleva al descubrimiento de la creatividad y vuelve a los sujetos capaces y seguros de aportar iniciativas, sugerencias y acciones transformadoras de su realidad cotidiana con alto sentido de identidad y pertinencia. 
La construccion social de valores, saberes y habilidades, contribuye al incremento de la conciencia social y a la responsabilidad en el ejercicio de los deberes ciudadanos, el pensarse como sujetos de derechos y deberes ha implicado asumir los valores éticos y políticos en la dignificación de sus propias vidas.

Desde la perspectiva de la Pedagogía crítica, si los maestros no desarrollan el pensamiento reflexivo y el análisis problematizador de su tarea de "enseñar", con base en su contexto singular no es factible promover el crecimiento autónomo y emancipador de los participantes en el proceso educativo. (POSADA, SIGNORI, 2009, pág. 51)

Por lo tanto, la participación no se da por el solo hecho de implementar tales espacios, sino por el trabajo pedagógico, de los procesos de planeaciòn, organizaciòn que se desarrollan de manera conjunta con los actores y con las organizaciones vinculadas y y de la reflexión contínua sobre la práctica. En suma, las estrategias pedagógicas que se promueven al interior de la Escuela, permiten las formación para la participacion, lo cual supone la construcción de personas capaces de pensar y actuar por sí mismas.

Hoy con certeza y sin lugar a dudas, La Escuela Popular Claretiana, sigue siendo una respuesta pedagógica alternativa para construir convivencia, en un país donde las condiciones económicas, culturales, históricas y políticas de cada contexto exigen nuevas demandas metodológicas; el proyecto se identifica con una ética liberadora y humanista, donde

los sueños son el motor de transformación y la educación liberadora como una herramienta para hacer posible, aunque sea posible una parte de lo imposible". , Y como lo expresó Freire y que continúa vigente “...una escuela que no se reinventa, que no asume su tarea como un acto político, no hace más que reproducir el orden social y condenar a la repetición tanto a los docentes como a los alumnos. ,(FREIRE, 2014, pág. 16).

Al momento surge los siguientes interrogantes: ¿Cuáles son los sueños de niños y niñas hoy? ¿Qué escuela se necesita reinventar hoy? ¿Cuál debe ser el rol del maestro? ¿Qué políticas públicas se requieren para fortalecer la experiencia pedagógica alternativa y la construcción de ,ciudadanía? 


\section{REFERENCIAS}

Federación Colombiana de educadores, FECODE, E.T. Declaración final del Congreso Pedagógico Nacional, Educación y cultura, Memorias, Bogotá, separata especial, p. 9-10, agosto. 1987.

FREINET, C, Por una educación del pueblo, Cuadernos de Educación, n. 49-50, Laboratorio Educativo, Caracas, 1977

FREIRE, P. Política y Educación. Editorial Siglo XXI Editores. México, 1997

FREIRE, P. Pedagogía de los sueños posibles. Por qué docentes y alumnos necesitan reinventarse en cada momento de la historia. editora Villa das Letras. Siglo Veintiuno Editores Argentina. S.A, 2015.

POSADA, E.T. La escuela Popular Claretiana: Una Experiencia de Innovación Educativa, Revista de Educación y Cultura. Memorias, Bogotá Colombia, Separata especial, p. 128-131, octubre. 1987

POSADA, SIGNORI, Filodehambre, una experiencia popular de innovación educativa. Editorial Presencia Ltda. Bogotá,1987.

POSADA, L, SIGNORI, A. Escuela Popular Claretiana. La participación en la escuela. Entretejiendo pasado, presente y futuro. Dimensión Educativa. Bogotá, 2009.

PROYECTO EDUCATIVO INSTITUCIONAL (PEI). Escuela Popular Claretiana, Neiva, 1997. 143p. 


\section{RESUMEN}

Este artículo expone el origen, fundamentación teórica, así como las estrategias de los procesos de participación y convivencia del proyecto educativo escolar, resume los avances de la Escuela Popular Claretiana, el balance, los factores que han posibilitado la implementación de la propuesta, las dificultades, el impacto y las conclusiones. La experiencia se desarrolla en Neiva-Colombia, a través de un enfoque de la Educación Popular, como respuesta al conflicto político militar, integra aspectos socioculturales, pedagógicos y didácticos; a través de la acción cooperativa, estrategias de participación, autogestión comunitaria y la creación de ambientes pedagógicos. La escuela tiene como propósito construir convivencia, potenciar los liderazgos, reafirmar la identidad cultural y el compromiso de las personas en la construcción de ciudadanía.

Palabras claves: Organización cooperativa, Ambientes, Ciudadanía.

\section{BUILDING COEXISTENCE IN A COUNTRY IN THE MIDDLE OF THE CONFLICT: AN ALTERNATIVE PEDAGOGICAL EXPERIENCE}

\section{ABSTRACT}

This article exposes the origin, theoretical foundation, as well as the strategies of the processes of participation and coexistence of the school educational project, summarizes the progress of the Claretian Popular School, the balance, the factors that have made possible the implementation of the proposal, difficulties, the impact and conclusions.

The experience is developed in Neiva-Colombia, through an approach on Popular Education, in response to military political conflict, integrating socio-cultural, pedagogical and didactic aspects; through cooperative organization, participation strategies, community self-management and the creation of pedagogical environments. The school aims to build coexistence, strengthen leadership, reaffirm the cultural identity and commitment of the subjects in the construction of citizenship.

Keywords: Cooperative organization, Environments, Citizenship.

\section{RESUMO}

Este artigo expõe a origem, fundamentação teórica, assim como as estratégias dos processos de participação e convivência do projeto educativo escolar. Resume os avances da Escola Popular Claretiana, o balanço, os fatores que tornaram possível a implementação da proposta, as dificuldades, o impacto e as conclusões.

A experiência se desenvolve em Neiva - Colômbia, através de um enfoque da Educação Popular, como resposta ao conflito político-militar, integra aspectos socioculturais, pedagógicos e didáticos; através da ação cooperativa, estratégias de participação, autogestão comunitária e a criação de ambientes pedagógicos. A escola tem como propósito construir convivência, potenciar as lideranças, reafirmar a identidade cultural e o compromisso das pessoas na construção de cidadania.

Palavras chave: Ação Cooperativa, Ambientes, Cidadania 